Supplement of Biogeosciences, 15, 5093-5111, 2018

https://doi.org/10.5194/bg-15-5093-2018-supplement

(C) Author(s) 2018. This work is distributed under

the Creative Commons Attribution 4.0 License.

(c) (1)

Supplement of

\title{
Modulation of the vertical particle transfer efficiency in the oxygen minimum zone off Peru
}

Marine Bretagnon et al.

Correspondence to: Marine Bretagnon (marine.bretagnon@legos.obs-mip.fr)

The copyright of individual parts of the supplement might differ from the CC BY 4.0 License. 


\section{Tables captions}

Tab. S1: Particles fluxes and their transfer efficiencies (T) according to the sampling dates for both AMOP1 $A M O P_{\text {summer }}$ (denoted AMOP1) and AMOP winter-spring (denoted AMOP2) datasets.

$T$ is determined from $\%$ Flux ${ }_{149 m} /$ Flux $_{34 m}$ and its uncertainty from logarithmic expansion calculation, and $b$ coefficient from the Martin's curves theory (Suess, 1980; Martin et al., 1987). Italic and non-italic values correspond to the fluxes at $34 \mathrm{~m}$ and $149 \mathrm{~m}$, respectively. On the last lines of the table, bold colored values in red, yellow and blue correspond to particles fluxes averaged values for low, intermediate and high $T_{\text {eff }}$ ranges, respectively, with the relative standard deviation between samples ( \pm SD\%). Analysis accuracy on the particles total mass fluxes is $\pm 3 \%$, inducing an absolute uncertainty on their vertical transfer efficiency estimated from a logarithmic expansion of $\pm 6 \%$ (cf. Methods).

Tab. S2: Organic elemental fluxes and their transfer efficiencies according to the sampling dates for both $A M O P 1$ AMOP summer $_{\text {(denoted AMOP1) and AMOP }}$ winter-spring $($ denoted AMOP2) datasets.

$a), b), c$ ) and d) POC, PON, POP and BSi fluxes in mg. $m^{-2} \cdot d^{-1}$, mmol. $m^{-2} \cdot d^{-1}$ and mol\% (on POC $+P O N+P O P+B S i$ ), and their transfer efficiency in \% ( $T_{\text {eff, }} T_{\text {effPoN }}$; $c$. Table 1 caption for calculation) in terms of Particulate Organic Carbon (POC), Nitrogen (PON), Phosphorus (POP) and Biogenic Silica (BSi) according to the sampling dates for both $A M O P_{\text {summer }}$ (denoted $A M O P$ ) and $A M O P_{\text {winter-spring }}$ (denoted $A M O P 2$ ) datasets.

For $a$ ), $b$ ), c) and d), italic and non-italic values correspond to the fluxes at $34 \mathrm{~m}$ and $149 \mathrm{~m}$, respectively. On the last lines of the table, bold values correspond to particles fluxes averaged values for low, intermediate and high $T_{\text {eff }}$ ranges, with the relative standard deviation between samples ( $\pm S D \%)$. Analysis accuracies on the elementary fluxes are $\pm 0.2 \%$ for both POC and PON, $\pm 3 \%$ for POP, and $\pm 5 \%$ for BSi, inducing an absolute uncertainty of $\pm 0.2 \%$ $\left(T_{\text {eff }}\right), \pm 0.2 \%\left(T_{\text {effPON }}\right), \pm 3 \%\left(T_{\text {effPOP }}\right)$ and $\pm 5 \%\left(T_{\text {effBSi }}\right)$ on the transfer efficiency (cf. Methods).

Tab. S3: Molar ratios and their transfer efficiencies according to the sampling dates for both AMOP1 $A M O P_{\text {summer }}($ denoted $A M O P 1)$ and AMOP ${ }_{\text {winter-spring }}$ (denoted AMOP2) datasets.

a) Values of elementary ratios $(C: N, C: P$ and $N: P)$ and transfer efficiency of these ratios in $\%\left(T_{\text {effC:N, }}, T_{\text {effC:P }}\right.$, and $T_{\text {effN:P; }}$ cf. Table 1 caption for calculation) according to the sampling dates for both AMOP summer $_{\text {(denoted AMOP1) }}$ and $A M O P_{\text {winter-spring }}($ denoted $A M O P 2)$ datasets.

b) Values of elementary ratios (Si:C, Si:N and Si:P) and transfer efficiency of these ratios in $\%\left(T_{\text {effSi:N, }} T_{\text {effsi:N }}\right.$ and $T_{\text {effSi:P; }}$ cf. Table 1 caption for calculation) according to the sampling dates for both AMOP summer $_{\text {(denoted AMOP1) }}$ and $A M O P_{\text {winter-spring }}$ (denoted AMOP2) datasets.

For $a$ ) and b), italic and non-italic values correspond to the fluxes at $34 \mathrm{~m}$ and $149 \mathrm{~m}$, respectively. On the last lines of the table, bold values correspond to particles fluxes averaged values for low, intermediate and high $T_{\text {eff }}$ ranges, with the relative standard deviation between samples $( \pm S D \%)$. Analysis accuracies on the elementary ratios are \pm 0.4, 3.2, 3.2\% and \pm 5.2, 5.2, 8\% for C:N, C:P and N:P and for Si:C, Si:N and Si:P, respectively, inducing an absolute uncertainty of $\pm 0.9 \%\left(T_{\text {effC:N }}\right), \pm 6.3 \%\left(T_{\text {effC:P }}\right), \pm 6 \%\left(T_{\text {effN:P }}\right)$, and of $\pm 12 \%\left(T_{\text {effSi:C }}\right), \pm 13.4 \%\left(T_{\text {effSi:N }}\right)$ and $\pm 19 \%\left(T_{\text {effsi:P}}\right)$ on the transfer efficiency cf. Methods. Classical (reference) molar ratios have been reported on the second lines from Redfield et al. (1963) and Brezinski (1985).

Tab. S4: Isotopic fluxes and their transfer efficiencies according to the sampling dates for both AMOP1

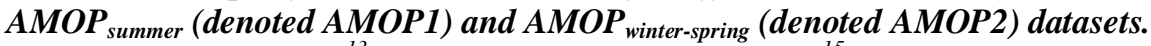

Carbon isotopic ratio $\left(\delta^{13} C\right)$ and nitrogen isotopic ratio $\left(\delta^{15} N\right)$ fluxes in \%o and their transfer efficiencies in $\%\left(T_{\text {effli3C }}\right.$ and $T_{\text {eff } 15 N}$; cf. Table 1 caption for calculation) according to the sampling dates for both AMOP summer (denoted $A M O P 1)$ and $A M O P_{\text {winter-spring }}$ (denoted $A M O P 2$ ) datasets. Italic and non-italic values correspond to the fluxes at 34 $m$ and $149 \mathrm{~m}$, respectively. On the last lines of the table, bold values correspond to particles fluxes averaged values for low, intermediate and high $T_{\text {eff }}$ ranges, with the relative standard deviation between samples $( \pm S D \%)$. Analysis accuracies on the isotopic values are $\pm 0.006 \%$ for $\delta^{13} \mathrm{C}$ and $\pm 0.007 \% \mathrm{for} \delta^{15} \mathrm{~N}$, inducing an absolute uncertainty of $\pm 0.06 \%\left(T_{\text {effl3C }}\right)$ and $\pm 0.26 \%\left(T_{\text {eff15N }}\right)$ on the transfer efficiency (cf. Methods). 
Tab. S5: Inorganic calcium carbonate flux and its transfer efficiencies according to the sampling dates for both AMOP1 AMOP summer $_{\text {(denoted AMOP1) and AMOP }}$ winter-spring (denoted AMOP2) datasets.

Inorganic calcium carbonate $\left(\mathrm{CaCO}_{3}\right)$ fluxes in $\mathrm{mgCa}^{-2} \cdot \mathrm{d}^{-1}$ and in \% of the total mass flux, and its transfer efficiency in \% ( $T_{\text {effCaCO3}}$; $c f$. Table 1 caption for calculation) according to the sampling dates for both AMOP summer (denoted $A M O P 1)$ and $A M O P_{\text {winter-spring }}($ denoted AMOP2) datasets. Italic and non-italic values correspond to the fluxes at $34 \mathrm{~m}$ and $149 \mathrm{~m}$, respectively. On the last lines of the table, bold values correspond to particles fluxes averaged values for low, intermediate and high $T_{\text {eff }}$ ranges, with the relative standard deviation between samples $( \pm S D \%)$. Analysis accuracies on the elementary fluxes are $\pm 3 \%$ for $\mathrm{CaCO}_{3}$, inducing an absolute uncertainty of $\pm 3 \%$ on the transfer efficiency $\left(T_{\text {effCaCO3}} ;\right.$ cf. Methods). 


\begin{tabular}{|c|c|c|c|c|c|c|}
\hline \multirow{3}{*}{ Sample name } & \multicolumn{2}{|c|}{ Date in 2013} & \multirow{2}{*}{\multicolumn{2}{|c|}{$\begin{array}{c}\text { TOTAL MASS FLUX } \\
\text { mg.m } \mathbf{m}^{-2} \cdot \mathrm{d}^{-1}\end{array}$}} & \multirow{3}{*}{$\begin{array}{c}\text { Transfer } \\
\text { efficiency } \\
\% \\
\text { T }\end{array}$} & \multirow{3}{*}{$\begin{array}{c}\text { Error bal } \\
\text { on } \mathbf{T} \\
\%\end{array}$} \\
\hline & \multirow[t]{2}{*}{ Start } & \multirow[t]{2}{*}{ End } & & & & \\
\hline & & & $34 m$ & $149 \mathrm{~m}$ & & \\
\hline AMOP1-S1 & $06 / 01$ & $13 / 01$ & 486.9 & 474.6 & 97 & \pm 1.2 \\
\hline AMOP1-S2 & $13 / 01$ & $20 / 01$ & 630.8 & 336.9 & 53 & \pm 0.7 \\
\hline AMOP1-S3 & $20 / 01$ & $27 / 01$ & 748.3 & 419.3 & 56 & \pm 0.6 \\
\hline AMOP1-S4 & $27 / 01$ & $03 / 02$ & 667.1 & 70.4 & 11 & \pm 0.5 \\
\hline AMOP1-S5 & $03 / 02$ & $10 / 02$ & 431.9 & 165.4 & 38 & \pm 1.0 \\
\hline AMOP1-S6 & $10 / 02$ & $17 / 02$ & 655.2 & 164.2 & 25 & \pm 0.6 \\
\hline AMOP1-S7 & $17 / 02$ & $24 / 02$ & 256.9 & 118.4 & 46 & \pm 1.7 \\
\hline AMOP1-S8 & $24 / 02$ & $03 / 03$ & 383.7 & 76.5 & 20 & \pm 0.9 \\
\hline AMOP1-S9 & $03 / 03$ & $10 / 03$ & 386.8 & 127.5 & 33 & \pm 1.0 \\
\hline AMOP1-S10 & $10 / 03$ & $17 / 03$ & 181.8 & 65.8 & 36 & \pm 2.2 \\
\hline AMOP1-S11 & $17 / 03$ & $24 / 03$ & 65.6 & 38.2 & 58 & \pm 7.2 \\
\hline AMOP1-S12 & $24 / 03$ & $31 / 03$ & 227.0 & 73.8 & 33 & \pm 1.8 \\
\hline AMOP2-S1 & $28 / 06$ & $09 / 07$ & 260.5 & 488.0 & 187 & \pm 3.3 \\
\hline AMOP2-S2 & 09/07 & $20 / 07$ & 235.4 & 154.3 & 66 & \pm 2.1 \\
\hline AMOP2-S3 & $20 / 07$ & $31 / 07$ & 81.9 & 123.8 & 151 & \pm 9.2 \\
\hline AMOP2-S4 & $31 / 07$ & $11 / 08$ & 83.0 & 25.3 & 30 & \pm 4.7 \\
\hline AMOP2-S5 & 11/08 & $22 / 08$ & 82.2 & 82.2 & 100 & \pm 7.3 \\
\hline AMOP2-S6 & $22 / 08$ & $02 / 09$ & 39.0 & 78.0 & 200 & \pm 23.1 \\
\hline AMOP2-S7 & $02 / 09$ & $13 / 09$ & 4647.1 & 48.6 & 1 & \pm 0.1 \\
\hline AMOP2-S8 & $13 / 09$ & 24/09 & 2998.3 & 18.9 & 1 & \pm 0.1 \\
\hline AMOP2-S9 & $24 / 09$ & $05 / 10$ & 1228.5 & 33.9 & 3 & \pm 0.3 \\
\hline AMOP2-S10 & $05 / 10$ & $16 / 10$ & 814.9 & 47.0 & 6 & \pm 0.4 \\
\hline AMOP2-S11 & $16 / 10$ & 27/10 & 877.2 & 30.8 & 4 & \pm 0.4 \\
\hline AMOP2-S12 & $27 / 10$ & 07/11 & 484.5 & 12.1 & 3 & \pm 0.6 \\
\hline & \multirow{2}{*}{\multicolumn{2}{|c|}{ High Teff }} & 328.6 & 278.1 & 81 & \\
\hline & & & $( \pm 69 \%)$ & $( \pm 79 \%)$ & $( \pm 80 \%)$ & \\
\hline & \multirow{2}{*}{\multicolumn{2}{|c|}{ Intermediate Teff }} & 408.6 & 144.9 & 49 & \\
\hline & & & $( \pm 61 \%)$ & $( \pm 77 \%)$ & $( \pm 85 \%)$ & \\
\hline & \multicolumn{2}{|c|}{ Low Teff } & $\begin{array}{c}1767.5 \\
( \pm 97 \%) \\
\end{array}$ & $\begin{array}{c}42.9 \\
( \pm 48 \%) \\
\end{array}$ & $\begin{array}{c}36 \\
( \pm 226 \%)\end{array}$ & \\
\hline
\end{tabular}

Table S1 


\begin{tabular}{|c|c|c|c|c|c|c|c|c|c|}
\hline \multirow{3}{*}{ a) $\begin{array}{c}\text { Sample } \\
\text { name }\end{array}$} & \multicolumn{2}{|c|}{ Date in 2013} & \multicolumn{6}{|c|}{ POC flux } & \multirow{3}{*}{$\begin{array}{c}\begin{array}{c}\text { Transfer } \\
\text { efficiency }\end{array} \\
\% \\
\mathrm{~T}_{\text {eff }}\end{array}$} \\
\hline & Start & End & $\operatorname{mgC} \cdot m^{-2} \cdot d^{-1}$ & $\operatorname{mmolC} \cdot \mathrm{m}^{-2} \cdot \mathrm{d}^{-1}$ & $\%$ molC & $\operatorname{mgC} \cdot m^{-2} \cdot d^{-1}$ & $\underset{1}{\operatorname{mmolC}} \cdot \mathrm{m}^{-2} \cdot \mathrm{d}^{-}$ & $\%$ molC & \\
\hline & & & \multicolumn{3}{|c|}{$34 \mathrm{~m}$} & \multicolumn{3}{|c|}{$149 \mathrm{~m}$} & \\
\hline AMOP1-S1 & $06 / 01$ & $13 / 01$ & 139.4 & 11.6 & 71 & 98.6 & 8.2 & 62 & 71 \\
\hline AMOP1-S2 & $13 / 01$ & $20 / 01$ & 127.9 & 10.7 & 66 & 70.7 & 5.9 & 60 & 55 \\
\hline AMOP1-S3 & $20 / 01$ & 27/01 & 149.5 & 12.5 & 64 & 85.9 & 7.2 & 62 & 57 \\
\hline AMOP1-S4 & $27 / 01$ & 03/02 & 92.2 & 7.7 & 69 & 22.1 & 1.8 & 65 & 24 \\
\hline AMOP1-S5 & $03 / 02$ & $10 / 02$ & 107.4 & 9.0 & 68 & 45.5 & 3.8 & 65 & 42 \\
\hline AMOP1-S6 & $10 / 02$ & $17 / 02$ & 132.7 & 11.1 & 64 & 41.5 & 3.5 & 61 & 31 \\
\hline AMOP1-S7 & $17 / 02$ & $24 / 02$ & 41.2 & 3.4 & 49 & 18.0 & 1.5 & 46 & 44 \\
\hline AMOP1-S8 & $24 / 02$ & $\mathbf{0 3 / 0 3}$ & 86.3 & 7.2 & 63 & 20.4 & 1.7 & 63 & 24 \\
\hline AMOP1-S9 & $03 / 03$ & $10 / 03$ & 109.7 & 9.1 & 72 & 37.9 & 3.2 & 70 & 35 \\
\hline AMOP1-S10 & $10 / 03$ & $17 / 03$ & 51.3 & 4.3 & 73 & 19.2 & 1.6 & 72 & 38 \\
\hline AMOP1-S11 & $17 / 03$ & $24 / 03$ & 20.3 & 1.7 & 74 & 11.6 & 1.0 & 76 & 57 \\
\hline AMOP1-S12 & 24/03 & $31 / 03$ & 54.1 & 4.5 & 65 & 20.1 & 1.7 & 65 & 37 \\
\hline AMOP2-S1 & 28/06 & 09/07 & 41.2 & 3.4 & 49 & 55.5 & 4.6 & 42 & 135 \\
\hline AMOP2-S2 & 09/07 & $20 / 07$ & 37.1 & 3.1 & 48 & 21.1 & 1.8 & 46 & 57 \\
\hline AMOP2-S3 & $20 / 07$ & 31/07 & 17.2 & 1.4 & 53 & 18.2 & 1.5 & 48 & 106 \\
\hline AMOP2-S4 & $31 / 07$ & $11 / 08$ & 19.4 & 1.6 & 65 & 6.2 & 0.5 & 69 & 32 \\
\hline AMOP2-S5 & 11/08 & $22 / 08$ & 17.9 & 1.5 & 56 & 12.2 & 1.0 & 47 & 68 \\
\hline AMOP2-S6 & $22 / 08$ & $02 / 09$ & 6.4 & 0.5 & 51 & 9.6 & 0.8 & 47 & 149 \\
\hline AMOP2-S7 & $02 / 09$ & $13 / 09$ & 470.5 & 39.2 & 41 & 8.3 & 0.7 & 59 & 2 \\
\hline AMOP2-S8 & $13 / 09$ & $24 / 09$ & 395.1 & 32.9 & 48 & 4.9 & 0.4 & 63 & 1 \\
\hline AMOP2-S9 & 24/09 & $05 / 10$ & 172.9 & 14.4 & 56 & 7.2 & 0.6 & 51 & 4 \\
\hline AMOP2-S10 & $05 / 10$ & $16 / 10$ & 135.0 & 11.3 & 58 & 7.0 & 0.6 & 40 & 5 \\
\hline AMOP2-S11 & $16 / 10$ & $27 / 10$ & 180.9 & 15.1 & 60 & 4.8 & 0.4 & 62 & 3 \\
\hline AMOP2-S12 & $27 / 10$ & $07 / 11$ & 83.0 & 6.9 & 63 & 3.0 & 0.3 & 71 & 4 \\
\hline & \multicolumn{2}{|c|}{ High Teff } & $\begin{array}{c}70.5 \\
( \pm 89 \%)\end{array}$ & $\begin{array}{c}5.9 \\
( \pm 89 \%)\end{array}$ & $\begin{array}{c}61 \\
( \pm 16 \%)\end{array}$ & $\begin{array}{c}40.3 \\
( \pm 88 \%)\end{array}$ & $\begin{array}{c}3.4 \\
( \pm 88 \%)\end{array}$ & $\begin{array}{c}58 \\
( \pm 21 \%)\end{array}$ & $\begin{array}{c}59 \\
( \pm 9 \%)\end{array}$ \\
\hline & \multirow{2}{*}{\multicolumn{2}{|c|}{$\begin{array}{c}\text { Intermediate } \\
\text { Teff }\end{array}$}} & 77.1 & 6.4 & 65 & 25.7 & 2.1 & 64 & 34 \\
\hline & & & $( \pm 49 \%)$ & $( \pm 49 \%)$ & $( \pm 11 \%)$ & $( \pm 50 \%)$ & $( \pm 50 \%)$ & $( \pm 12 \%)$ & $( \pm 21 \%)$ \\
\hline & \multicolumn{2}{|c|}{ Low Teff } & $\begin{array}{l}239.6 \\
( \pm 65 \%)\end{array}$ & $\begin{array}{l}20,0 \\
( \pm 65 \%)\end{array}$ & $\begin{array}{c}54 \\
( \pm 15 \%)\end{array}$ & $\begin{array}{c}5.9 \\
( \pm 33 \%)\end{array}$ & $\begin{array}{c}0.5 \\
( \pm 33 \%)\end{array}$ & $\begin{array}{c}\mathbf{5 7} \\
( \pm \mathbf{1 8 \%} \%\end{array}$ & $\begin{array}{c}3 \\
( \pm 48 \%)\end{array}$ \\
\hline
\end{tabular}

Table S2a 


\begin{tabular}{|c|c|c|c|c|c|c|c|c|c|}
\hline \multirow{3}{*}{ b) $\begin{array}{c}\text { Sample } \\
\text { name }\end{array}$} & \multicolumn{2}{|c|}{ Date in 2013} & \multicolumn{6}{|c|}{ PON flux } & \multirow{3}{*}{$\begin{array}{c}\begin{array}{c}\text { Transfer } \\
\text { efficiency }\end{array} \\
\% \\
\mathrm{~T}\end{array}$} \\
\hline & Start & End & $\mathrm{mgN} \cdot \mathrm{m}^{-2} \cdot \mathrm{d}^{-1}$ & mmolN.m ${ }^{-2} \cdot d^{-1}$ & $\%$ molN & $\mathrm{mgN} \cdot \mathrm{m}^{-2} \cdot \mathrm{d}^{-1}$ & mmolN.m ${ }^{-2} \cdot d^{-1}$ & $\%$ molN & \\
\hline & & & \multicolumn{3}{|c|}{$34 m$} & \multicolumn{3}{|c|}{$149 \mathrm{~m}$} & \\
\hline AMOP1-S1 & $06 / 01$ & $13 / 01$ & 23.6 & 1.7 & 10 & 15.7 & 1.1 & 8 & 67 \\
\hline AMOP1-S2 & $13 / 01$ & $20 / 01$ & 19.8 & 1.4 & 9 & 10.5 & 0.8 & 8 & 53 \\
\hline AMOP1-S3 & $20 / 01$ & $27 / 01$ & 20.8 & 1.5 & 8 & 11.5 & 0.8 & 7 & 55 \\
\hline AMOP1-S4 & $27 / 01$ & $03 / 02$ & 14.3 & 1.0 & 9 & 3.6 & 0.3 & 9 & 25 \\
\hline AMOP1-S5 & $03 / 02$ & $10 / 02$ & 14.3 & 1.0 & 8 & 6.3 & 0.5 & 8 & 44 \\
\hline AMOP1-S6 & $10 / 02$ & 17/02 & 17.3 & 1.2 & 7 & 7.1 & 0.5 & 9 & 41 \\
\hline AMOP1-S7 & $17 / 02$ & $24 / 02$ & 5.7 & 0.4 & 6 & 2.2 & 0.2 & 5 & 39 \\
\hline AMOP1-S8 & $24 / 02$ & $\mathbf{0 3 / 0 3}$ & 12.0 & 0.9 & 7 & 2.4 & 0.2 & 6 & 20 \\
\hline AMOP1-S9 & $03 / 03$ & $10 / 03$ & 15.5 & 1.1 & 9 & 5.0 & 0.4 & 8 & 32 \\
\hline AMOP1-S10 & $10 / 03$ & $17 / 03$ & 7.4 & 0.5 & 9 & 2.4 & 0.2 & 8 & 32 \\
\hline AMOP1-S11 & $17 / 03$ & $24 / 03$ & 2.7 & 0.2 & 8 & 1.8 & 0.1 & 10 & 65 \\
\hline AMOP1-S12 & $24 / 03$ & $31 / 03$ & 6.2 & 0.4 & 6 & 2.9 & 0.2 & 8 & 47 \\
\hline AMOP2-S1 & $28 / 06$ & 09/07 & 6.4 & 0.5 & 7 & 7.1 & 0.5 & 5 & 110 \\
\hline AMOP2-S2 & 09/07 & $20 / 07$ & 6.0 & 0.4 & 7 & 2.8 & 0.2 & 5 & 46 \\
\hline AMOP2-S3 & $20 / 07$ & 31/07 & 2.9 & 0.2 & 8 & 2.5 & 0.2 & 6 & 84 \\
\hline AMOP2-S4 & $31 / 07$ & $11 / 08$ & 3.8 & 0.3 & 11 & 1.0 & 0.1 & 10 & 26 \\
\hline AMOP2-S5 & 11/08 & $22 / 08$ & 3.4 & 0.2 & 9 & 1.7 & 0.1 & 6 & 51 \\
\hline AMOP2-S6 & $22 / 08$ & $02 / 09$ & 1.1 & 0.1 & 8 & 1.4 & 0.1 & 6 & 123 \\
\hline AMOP2-S7 & $02 / 09$ & $13 / 09$ & 77.3 & 5.5 & 6 & 1.1 & 0.1 & 7 & 1 \\
\hline AMOP2-S8 & $13 / 09$ & $24 / 09$ & 67.3 & 4.8 & 7 & 0.8 & 0.1 & 8 & 1 \\
\hline AMOP2-S9 & 24/09 & $\mathbf{0 5} / 10$ & 26.0 & 1.9 & 7 & 1.0 & 0.1 & 6 & 4 \\
\hline AMOP2-S10 & $05 / 10$ & $16 / 10$ & 23.6 & 1.7 & 9 & 0.9 & 0.1 & 4 & 4 \\
\hline AMOP2-S11 & $16 / 10$ & $27 / 10$ & 36.7 & 2.6 & 10 & 0.9 & 0.1 & 10 & 3 \\
\hline AMOP2-S12 & $27 / 10$ & 07/11 & 16.5 & 1.2 & 11 & 0.7 & 0.0 & 14 & 4 \\
\hline & \multirow{2}{*}{\multicolumn{2}{|c|}{ High Teff }} & 10.6 & 0.8 & 8 & 5.7 & 0.4 & 7 & 54 \\
\hline & & & $( \pm 85 \%)$ & $( \pm 85 \%)$ & $( \pm 11 \%)$ & $( \pm 87 \%)$ & $( \pm 87 \%)$ & $( \pm 26 \%)$ & $( \pm 13 \%)$ \\
\hline & \multirow{2}{*}{\multicolumn{2}{|c|}{$\begin{array}{c}\text { Intermediate } \\
\text { Teff }\end{array}$}} & 10.7 & 0.8 & 8 & 3.7 & 0.3 & 8 & 34 \\
\hline & & & $( \pm 46 \%)$ & $( \pm 46 \%)$ & $( \pm 20 \%)$ & $( \pm 56 \%)$ & $( \pm 56 \%)$ & $( \pm 19 \%)$ & $( \pm 27 \%)$ \\
\hline & \multirow{2}{*}{\multicolumn{2}{|c|}{ Low Teff }} & 41.2 & 2.9 & 8 & 0.9 & 0.1 & 8 & 3 \\
\hline & & & $( \pm 61 \%)$ & $( \pm 61 \%)$ & $( \pm 24 \%)$ & $( \pm 18 \%)$ & $( \pm 18 \%)$ & $( \pm 41 \%)$ & $( \pm 47 \%)$ \\
\hline
\end{tabular}

Table S2b 


\begin{tabular}{|c|c|c|c|c|c|c|c|c|c|}
\hline \multirow{3}{*}{$\begin{array}{l}\text { Sample } \\
\text { name }\end{array}$} & \multicolumn{2}{|c|}{ Date in 2013} & \multicolumn{6}{|c|}{ POP flux } & \multirow{3}{*}{$\begin{array}{c}\begin{array}{c}\text { Transfer } \\
\text { efficiency }\end{array} \\
\% \\
\mathrm{~T}_{\text {effPOP }}\end{array}$} \\
\hline & Start & End & $\mathrm{mgP} \cdot \mathrm{m}^{-2} \cdot \mathrm{d}^{-1}$ & mmolP.m $m^{-2} \cdot d^{-1}$ & \% molP & $\operatorname{mgP} \cdot m^{-2} \cdot d^{-1}$ & mmolP. $\mathbf{m}^{-2} \cdot d^{-1}$ & $\%$ molP & \\
\hline & & & \multicolumn{3}{|c|}{$34 m$} & \multicolumn{3}{|c|}{$149 \mathrm{~m}$} & \\
\hline AMOP1-S1 & $06 / 01$ & $13 / 01$ & 17.3 & 0.6 & 4 & 5.6 & 0.2 & 1 & 32 \\
\hline AMOP1-S2 & $13 / 01$ & 20/01 & 7.1 & 0.2 & 1 & 2.4 & 0.1 & 1 & 34 \\
\hline AMOP1-S3 & $20 / 01$ & 27/01 & 5.9 & 0.2 & 1 & 2.1 & 0.1 & 1 & 35 \\
\hline AMOP1-S4 & 27/01 & $03 / 02$ & 3.5 & 0.1 & 1 & 0.9 & 0.0 & 1 & 26 \\
\hline AMOP1-S5 & $03 / 02$ & $10 / 02$ & 4.7 & 0.2 & 1 & 1.3 & 0.0 & 1 & 27 \\
\hline AMOP1-S6 & $10 / 02$ & $17 / 02$ & 3.9 & 0.1 & 1 & 2.6 & 0.1 & 2 & 66 \\
\hline AMOP1-S7 & $17 / 02$ & $24 / 02$ & 2.2 & 0.1 & 1 & 0.9 & 0.0 & 1 & 40 \\
\hline AMOP1-S8 & $24 / 02$ & $03 / 03$ & 3.4 & 0.1 & 1 & 0.9 & 0.0 & 1 & 27 \\
\hline AMOP1-S9 & $03 / 03$ & $10 / 03$ & 6.4 & 0.2 & 2 & 1.6 & 0.1 & 1 & 25 \\
\hline AMOP1-S10 & $10 / 03$ & $17 / 03$ & 2.7 & 0.1 & 2 & 1.4 & 0.0 & 2 & 53 \\
\hline AMOP1-S11 & $17 / 03$ & $24 / 03$ & 1.0 & 0.0 & 1 & 0.5 & 0.0 & 1 & 45 \\
\hline AMOP1-S12 & $24 / 03$ & $31 / 03$ & 3.0 & 0.1 & 1 & 0.9 & 0.0 & 1 & 31 \\
\hline AMOP2-S1 & $28 / 06$ & 09/07 & 1.8 & 0.1 & 1 & 2.7 & 0.1 & 1 & 152 \\
\hline AMOP2-S2 & 09/07 & 20/07 & 2.0 & 0.1 & 1 & 1.6 & 0.1 & 1 & 80 \\
\hline AMOP2-S3 & $20 / 07$ & 31/07 & 0.8 & 0.0 & 1 & 0.8 & 0.0 & 1 & 104 \\
\hline AMOP2-S4 & $31 / 07$ & 11/08 & 3.7 & 0.1 & 5 & 0.4 & 0.0 & 2 & 12 \\
\hline AMOP2-S5 & 11/08 & $22 / 08$ & 0.5 & 0.0 & 1 & 0.5 & 0.0 & 1 & 107 \\
\hline AMOP2-S6 & $22 / 08$ & $02 / 09$ & 0.2 & 0.0 & 1 & 0.5 & 0.0 & 1 & 267 \\
\hline AMOP2-S7 & $02 / 09$ & $13 / 09$ & 9.2 & 0.3 & 0 & 0.2 & 0.0 & 1 & 3 \\
\hline AMOP2-S8 & $13 / 09$ & 24/09 & 8.3 & 0.3 & 0 & 0.1 & 0.0 & 1 & 2 \\
\hline AMOP2-S9 & $24 / 09$ & $05 / 10$ & 2.8 & 0.1 & 0 & 0.5 & 0.0 & 2 & 19 \\
\hline AMOP2-S10 & $05 / 10$ & $16 / 10$ & 2.2 & 0.1 & 0 & 0.6 & 0.0 & 1 & 25 \\
\hline AMOP2-S11 & $16 / 10$ & $27 / 10$ & 5.1 & 0.2 & 1 & 1.5 & 0.1 & 8 & 30 \\
\hline AMOP2-S12 & $27 / 10$ & 07/11 & 2.0 & 0.1 & 1 & 0.2 & 0.0 & 2 & 9 \\
\hline & \multirow{2}{*}{\multicolumn{2}{|c|}{ High Teff }} & 3.3 & 0.1 & 1 & 1.4 & 0.0 & 1 & 60 \\
\hline & & & $( \pm 91 \%)$ & $( \pm 91 \%)$ & $( \pm 32 \%)$ & $( \pm 63 \%)$ & $( \pm 63 \%)$ & $( \pm 32 \%)$ & $( \pm 53 \%)$ \\
\hline & \multirow{2}{*}{\multicolumn{2}{|c|}{$\begin{array}{c}\text { Intermediate } \\
\text { Teff }\end{array}$}} & 3.7 & 0.1 & 2 & 1.2 & 0.0 & 1 & 34 \\
\hline & & & $( \pm 33 \%)$ & $( \pm 33 \%)$ & $( \pm 79 \%)$ & $( \pm 50 \%)$ & $( \pm 50 \%)$ & $( \pm 36 \%)$ & $( \pm 48 \%)$ \\
\hline & \multirow{2}{*}{\multicolumn{2}{|c|}{ Low Teff }} & 4.9 & 0.2 & 0 & 0.5 & 0.0 & 2 & 15 \\
\hline & & & $( \pm 64 \%)$ & $( \pm 64 \%)$ & $( \pm 33 \%)$ & $( \pm 98 \%)$ & $( \pm 98 \%)$ & $( \pm 120 \%)$ & $( \pm 81 \%)$ \\
\hline
\end{tabular}

Table S2c 


\begin{tabular}{|c|c|c|c|c|c|c|c|c|c|}
\hline \multirow{3}{*}{$\begin{array}{l}\text { Sample } \\
\text { name }\end{array}$} & \multicolumn{2}{|c|}{ Date in 2013} & \multicolumn{6}{|c|}{ BSi flux } & \multirow{3}{*}{$\begin{array}{c}\text { Transfer } \\
\text { efficiency } \\
\% \\
\mathrm{~T}_{\text {effBSi }}\end{array}$} \\
\hline & Start & End & mgSi.m ${ }^{-2} \cdot d^{-1}$ & mmolSi.m ${ }^{-2} \cdot d^{-1}$ & $\%$ molSi & mgSi.m-2 $\cdot d^{-1}$ & mmolSi.m-2. $\mathrm{d}^{-1}$ & $\%$ molSi & \\
\hline & & & \multicolumn{3}{|c|}{$34 m$} & \multicolumn{3}{|c|}{$149 \mathrm{~m}$} & \\
\hline AMOP1-S1 & $06 / 01$ & $13 / 01$ & 69.3 & 2.5 & 15 & 105.6 & 3.8 & 28 & 152 \\
\hline AMOP1-S2 & $13 / 01$ & 20/01 & 110.6 & 3.9 & 24 & 85.5 & 3.1 & 31 & 77 \\
\hline AMOP1-S3 & $20 / 01$ & 27/01 & 151.8 & 5.4 & 28 & 97.4 & 3.5 & 30 & 64 \\
\hline AMOP1-S4 & $27 / 01$ & $03 / 02$ & 66.3 & 2.4 & 21 & 19.6 & 0.7 & 25 & 30 \\
\hline AMOP1-S5 & $03 / 02$ & $10 / 02$ & 84.5 & 3.0 & 23 & 43.1 & 1.5 & 26 & 51 \\
\hline AMOP1-S6 & $10 / 02$ & $17 / 02$ & 138.5 & 4.9 & 28 & 46.4 & 1.7 & 29 & 33 \\
\hline AMOP1-S7 & $17 / 02$ & $24 / 02$ & 84.9 & 3.0 & 44 & 44.1 & 1.6 & 48 & 52 \\
\hline AMOP1-S8 & $24 / 02$ & $03 / 03$ & 93.0 & 3.3 & 29 & 22.2 & 0.8 & 29 & 24 \\
\hline AMOP1-S9 & $03 / 03$ & $10 / 03$ & 61.8 & 2.2 & 17 & 26.2 & 0.9 & 21 & 42 \\
\hline AMOP1-S10 & $10 / 03$ & $17 / 03$ & 27.1 & 1.0 & 17 & 11.4 & 0.4 & 18 & 42 \\
\hline AMOP1-S11 & $17 / 03$ & $24 / 03$ & 10.3 & 0.4 & 16 & 4.8 & 0.2 & 13 & 47 \\
\hline AMOP1-S12 & $24 / 03$ & 31/03 & 51.7 & 1.8 & 27 & 18.2 & 0.7 & 25 & 35 \\
\hline AMOP2-S1 & 28/06 & 09/07 & 84.1 & 3.0 & 43 & 158.5 & 5.7 & 52 & 188 \\
\hline AMOP2-S2 & $09 / 07$ & 20/07 & 78.1 & 2.8 & 44 & 50.6 & 1.8 & 47 & 65 \\
\hline AMOP2-S3 & 20/07 & 31/07 & 28.7 & 1.0 & 38 & 39.9 & 1.4 & 45 & 139 \\
\hline AMOP2-S4 & 31/07 & $11 / 08$ & 13.5 & 0.5 & 19 & 4.1 & 0.1 & 20 & 30 \\
\hline AMOP2-S5 & $11 / 08$ & $22 / 08$ & 26.0 & 0.9 & 35 & 28.4 & 1.0 & 47 & 109 \\
\hline AMOP2-S6 & $22 / 08$ & $02 / 09$ & 12.1 & 0.4 & 41 & 21.6 & 0.8 & 46 & 178 \\
\hline AMOP2-S7 & $02 / 09$ & $13 / 09$ & 1394.8 & 49.8 & 53 & 11.2 & 0.4 & 34 & 1 \\
\hline AMOP2-S8 & $13 / 09$ & $24 / 09$ & 863.8 & 30.8 & 45 & 5.2 & 0.2 & 28 & 1 \\
\hline AMOP2-S9 & $24 / 09$ & $05 / 10$ & 256.4 & 9.2 & 36 & 13.5 & 0.5 & 41 & 5 \\
\hline AMOP2-S10 & $05 / 10$ & $16 / 10$ & 179.8 & 6.4 & 33 & 21.9 & 0.8 & 54 & 12 \\
\hline AMOP2-S11 & $16 / 10$ & 27/10 & 199.4 & 7.1 & 28 & 3.7 & 0.1 & 20 & 2 \\
\hline AMOP2-S12 & $27 / 10$ & 07/11 & 78.9 & 2.8 & 26 & 1.3 & 0.0 & 13 & 2 \\
\hline \multicolumn{3}{|c|}{ High Teff } & $\begin{array}{c}75.4 \\
( \pm 78 \%)\end{array}$ & $\begin{array}{c}2.7 \\
( \pm 78 \%)\end{array}$ & $\begin{array}{c}29 \\
( \pm 36 \%)\end{array}$ & $\begin{array}{c}53.3 \\
( \pm 72 \%)\end{array}$ & $\begin{array}{c}1.9 \\
( \pm 72 \%)\end{array}$ & $\begin{array}{c}34 \\
( \pm 41 \%)\end{array}$ & $\begin{array}{c}72 \\
( \pm 32 \%)\end{array}$ \\
\hline & \multirow{2}{*}{\multicolumn{2}{|c|}{$\begin{array}{c}\text { Intermediate } \\
\text { Teff }\end{array}$}} & 69.0 & 2.5 & 25 & 26.2 & 0.9 & 27 & 38 \\
\hline & & & $( \pm 54 \%)$ & $( \pm 54 \%)$ & $( \pm 33 \%)$ & $( \pm 58 \%)$ & $( \pm 58 \%)$ & $( \pm 33 \%)$ & $( \pm 26 \%)$ \\
\hline & \multicolumn{2}{|c|}{ Low Teff } & $\begin{array}{l}495.5 \\
( \pm 105 \%)\end{array}$ & $\begin{array}{c}17.7 \\
( \pm 105 \%)\end{array}$ & $\begin{array}{c}37 \\
( \pm 28 \%)\end{array}$ & $\begin{array}{c}9.5 \\
( \pm 81 \%)\end{array}$ & $\begin{array}{c}0.3 \\
( \pm 81 \%) \\
\end{array}$ & $\begin{array}{c}32 \\
( \pm 46 \%) \\
\end{array}$ & $\begin{array}{c}4 \\
( \pm 120 \%)\end{array}$ \\
\hline
\end{tabular}

Table S2d 


\begin{tabular}{|c|c|c|c|c|c|c|c|c|c|c|c|}
\hline \multirow{3}{*}{$\begin{array}{c}\text { a) } \\
\text { Sample } \\
\text { name }\end{array}$} & \multicolumn{2}{|c|}{ Date in 2013} & \multirow{2}{*}{\multicolumn{2}{|c|}{$\begin{array}{c}\mathrm{C}: \mathrm{N} \\
6.625\end{array}$}} & \multirow{3}{*}{$\begin{array}{c}\mathbf{T}_{\text {effC:N }} \\
\%\end{array}$} & \multicolumn{2}{|c|}{$\mathrm{C}: \mathbf{P}$} & \multirow{3}{*}{$\begin{array}{c}\mathbf{T}_{\text {effC:P }} \\
\%\end{array}$} & \multirow{2}{*}{\multicolumn{2}{|c|}{$\begin{array}{c}\mathbf{N}: \mathbf{P} \\
16\end{array}$}} & \multirow{3}{*}{$\begin{array}{c}\mathbf{T}_{\text {effN:P }} \\
\%\end{array}$} \\
\hline & \multirow[t]{2}{*}{ Start } & \multirow[t]{2}{*}{ End } & & & & & & & & & \\
\hline & & & $34 m$ & $149 \mathrm{~m}$ & & $34 m$ & $149 \mathrm{~m}$ & & $34 \mathrm{~m}$ & $149 \mathrm{~m}$ & \\
\hline AMOP1-S1 & $06 / 01$ & 13/01 & 6.89 & 7.31 & 106 & 20.83 & 45.75 & 220 & 3.02 & 6.26 & 207 \\
\hline AMOP1-S2 & 13/01 & $20 / 01$ & 7.52 & 7.83 & 104 & 46.48 & 75.02 & 161 & 6.18 & 9.58 & 155 \\
\hline AMOP1-S3 & $20 / 01$ & $27 / 01$ & 8.37 & 8.72 & 104 & 65.64 & 106.59 & 162 & 7.84 & 12.23 & 156 \\
\hline AMOP1-S4 & $27 / 01$ & 03/02 & 7.53 & 7.13 & 95 & 67.8 & 62.03 & 91 & 9.00 & 8.7 & 97 \\
\hline AMOP1-S5 & $03 / 02$ & $10 / 02$ & 8.78 & 8.36 & 95 & 58.99 & 92.12 & 156 & 6.72 & 11.02 & 164 \\
\hline AMOP1-S6 & $10 / 02$ & $17 / 02$ & 8.94 & 6.83 & 76 & 88.38 & 41.67 & 47 & 9.89 & 6.1 & 62 \\
\hline AMOP1-S7 & $17 / 02$ & $24 / 02$ & 8.48 & 9.55 & 113 & 48.6 & 52.87 & 109 & 5.73 & 5.53 & 97 \\
\hline AMOP1-S8 & 24/02 & $03 / 03$ & 8.39 & 10.03 & 120 & 66.48 & 58.57 & 88 & 7.93 & 5.84 & 74 \\
\hline AMOP1-S9 & $03 / 03$ & $10 / 03$ & 8.25 & 8.81 & 107 & 44.4 & 60.43 & 136 & 5.38 & 6.86 & 127 \\
\hline AMOP1-S10 & $10 / 03$ & $17 / 03$ & 8.09 & 9.39 & 116 & 49.11 & 34.94 & 71 & 6.07 & 3.72 & 61 \\
\hline AMOP1-S11 & $17 / 03$ & $24 / 03$ & 8.7 & 7.67 & 88 & 51.68 & 65.93 & 128 & 5.94 & 8.6 & 145 \\
\hline AMOP1-S12 & $24 / 03$ & $31 / 03$ & 10.11 & 8.01 & 79 & 46.78 & 55.48 & 119 & 4.63 & 6.93 & 150 \\
\hline AMOP2-S1 & 28/06 & 09/07 & 7.47 & 9.14 & 122 & 59.29 & 52.59 & 89 & 7.93 & 5.75 & 72 \\
\hline AMOP2-S2 & 09/07 & $20 / 07$ & 7.17 & 8.81 & 123 & 48.7 & 34.62 & 71 & 6.79 & 3.93 & 58 \\
\hline AMOP2-S3 & $20 / 07$ & 31/07 & 6.8 & 8.62 & 127 & 55.06 & 56.17 & 102 & 8.1 & 6.52 & 80 \\
\hline AMOP2-S4 & $31 / 07$ & $11 / 08$ & 5.91 & 7.13 & 121 & 13.49 & 36.27 & 269 & 2.28 & 5.09 & 223 \\
\hline AMOP2-S5 & $11 / 08$ & $22 / 08$ & 6.18 & 8.30 & 134 & 91.12 & 57.91 & 64 & 14.75 & 6.98 & 47 \\
\hline AMOP2-S6 & $22 / 08$ & $02 / 09$ & 6.62 & 7.99 & 121 & 96.15 & 53.67 & 56 & 14.52 & 6.72 & 46 \\
\hline AMOP2-S7 & $02 / 09$ & $13 / 09$ & 7.1 & 8.55 & 120 & 132.48 & 88.43 & 67 & 18.66 & 10.35 & 55 \\
\hline AMOP2-S8 & $13 / 09$ & $24 / 09$ & 6.85 & 7.53 & 110 & 123.45 & 88.11 & 71 & 18.02 & 11.7 & 65 \\
\hline AMOP2-S9 & 24/09 & $05 / 10$ & 7.75 & 8.05 & 104 & 159.21 & 34.67 & 22 & 20.53 & 4.31 & 21 \\
\hline AMOP2-S10 & $05 / 10$ & $16 / 10$ & 6.69 & 9.21 & 138 & 160.16 & 32.63 & 20 & 23.95 & 3.54 & 15 \\
\hline AMOP2-S11 & $16 / 10$ & $27 / 10$ & 5.75 & 5.95 & 103 & 90.7 & 8.1 & 9 & 15.78 & 1.36 & 9 \\
\hline AMOP2-S12 & $27 / 10$ & $07 / 11$ & 5.86 & 5.00 & 85 & 105.16 & 42.17 & 40 & 17.94 & 8.43 & 47 \\
\hline & Hig & eff & $\begin{array}{c}7.59 \\
( \pm 13 \%)\end{array}$ & $\begin{array}{l}8.26 \\
( \pm 6 \%)\end{array}$ & $\underset{( \pm 16 \%)}{111}$ & $\begin{array}{l}60.72 \\
( \pm 31 \%)\end{array}$ & $\begin{array}{l}\mathbf{6 8 . 0 1} \\
( \pm 39 \%)\end{array}$ & $\underset{( \pm 41 \%)}{117}$ & $\begin{array}{c}8.3 \\
( \pm 44 \%)\end{array}$ & $\begin{array}{c}\mathbf{8 . 2 6} \\
( \pm 37 \%)\end{array}$ & $\underset{( \pm 49 \%)}{112}$ \\
\hline & Interm & te Teff & $\begin{array}{c}8.27 \\
( \pm 14 \%)\end{array}$ & $\begin{array}{c}8.36 \\
( \pm 14 \%)\end{array}$ & $\begin{array}{c}102 \\
( \pm 16 \%)\end{array}$ & $\begin{array}{l}53.78 \\
( \pm 38 \%)\end{array}$ & $\begin{array}{l}54.93 \\
( \pm 32 \%)\end{array}$ & $\begin{array}{c}121 \\
( \pm 54 \%)\end{array}$ & $\begin{array}{c}6.4 \\
( \pm 36 \%)\end{array}$ & $\begin{array}{c}6.64 \\
( \pm 32 \%)\end{array}$ & $\begin{array}{c}117 \\
( \pm 46 \%)\end{array}$ \\
\hline & Lo & eff & $\begin{array}{c}6.67 \\
( \pm 11 \%)\end{array}$ & $\begin{array}{c}7.38 \\
( \pm 22 \%)\end{array}$ & $\begin{array}{c}110 \\
( \pm 16 \%)\end{array}$ & $\begin{array}{c}128.53 \\
( \pm 22 \%)\end{array}$ & $\begin{array}{l}49.02 \\
( \pm 66 \%)\end{array}$ & $\begin{array}{c}38 \\
( \pm 68 \%)\end{array}$ & $\begin{array}{l}19.15 \\
( \pm 15 \%)\end{array}$ & $\begin{array}{c}6.61 \\
( \pm 62 \%)\end{array}$ & $\begin{array}{c}35 \\
( \pm 67 \%)\end{array}$ \\
\hline
\end{tabular}

Table S3a 


\begin{tabular}{|c|c|c|c|c|c|c|c|c|c|c|c|}
\hline \multirow{3}{*}{$\begin{array}{c}\text { b) } \\
\text { Sample } \\
\text { name }\end{array}$} & \multicolumn{2}{|c|}{ Date in 2013} & \multirow{2}{*}{\multicolumn{2}{|c|}{$\begin{array}{l}\text { Si:C } \\
0.14\end{array}$}} & \multirow{3}{*}{$\begin{array}{c}\mathbf{T}_{\text {effSi:C }} \\
\%\end{array}$} & \multirow{2}{*}{\multicolumn{2}{|c|}{$\begin{array}{l}\text { Si:N } \\
0.94\end{array}$}} & \multirow{3}{*}{$\begin{array}{c}\mathbf{T}_{\text {effSi:N }} \\
\%\end{array}$} & \multirow{2}{*}{\multicolumn{2}{|c|}{$\begin{array}{c}\text { Si:P } \\
15\end{array}$}} & \multirow{3}{*}{$\begin{array}{c}\mathbf{T}_{\text {effSi:P }} \\
\%\end{array}$} \\
\hline & \multirow[t]{2}{*}{ Start } & \multirow[t]{2}{*}{ End } & & & & & & & & & \\
\hline & & & $34 m$ & $149 \mathrm{~m}$ & & $34 m$ & $149 \mathrm{~m}$ & & $34 \mathrm{~m}$ & $149 \mathrm{~m}$ & \\
\hline AMOP1-S1 & $06 / 01$ & $13 / 01$ & 0.21 & 0.46 & 215 & 1.46 & 3.34 & 228 & 4.42 & 20.92 & 473 \\
\hline AMOP1-S2 & $13 / 01$ & $20 / 01$ & 0.37 & 0.52 & 140 & 2.78 & 4.05 & 146 & 17.17 & 38.77 & 226 \\
\hline AMOP1-S3 & $20 / 01$ & $27 / 01$ & 0.43 & 0.48 & 112 & 3.63 & 4.22 & 116 & 28.49 & 51.65 & 181 \\
\hline AMOP1-S4 & $27 / 01$ & $03 / 02$ & 0.31 & 0.38 & 123 & 2.31 & 2.7 & 117 & 20.83 & 23.52 & 113 \\
\hline AMOP1-S5 & $03 / 02$ & $10 / 02$ & 0.34 & 0.41 & 121 & 2.95 & 3.39 & 115 & 19.84 & 37.33 & 188 \\
\hline AMOP1-S6 & $10 / 02$ & $17 / 02$ & 0.45 & 0.48 & 107 & 3.99 & 3.26 & 82 & 39.42 & 19.89 & 50 \\
\hline AMOP1-S7 & $17 / 02$ & $24 / 02$ & 0.88 & 1.05 & 119 & 7.47 & 10.00 & 134 & 42.83 & 55.34 & 129 \\
\hline AMOP1-S8 & $24 / 02$ & $03 / 03$ & 0.46 & 0.46 & 101 & 3.86 & 4.66 & 121 & 30.62 & 27.21 & 89 \\
\hline AMOP1-S9 & $03 / 03$ & $10 / 03$ & 0.24 & 0.3 & 123 & 1.99 & 2.61 & 131 & 10.68 & 17.87 & 167 \\
\hline AMOP1-S10 & $10 / 03$ & $17 / 03$ & 0.23 & 0.25 & 112 & 1.83 & 2.38 & 130 & 11.08 & 8.85 & 80 \\
\hline AMOP1-S11 & $17 / 03$ & $24 / 03$ & 0.22 & 0.18 & 82 & 1.89 & 1.36 & 72 & 11.23 & 11.73 & 104 \\
\hline AMOP1-S12 & $24 / 03$ & $31 / 03$ & 0.41 & 0.39 & 95 & 4.13 & 3.1 & 75 & 19.10 & 21.44 & 112 \\
\hline AMOP2-S1 & 28/06 & 09/07 & 0.87 & 1.22 & 140 & 6.52 & 11.16 & 171 & 51.71 & 64.17 & 124 \\
\hline AMOP2-S2 & 09/07 & $20 / 07$ & 0.9 & 1.02 & 114 & 6.46 & 9.01 & 139 & 43.88 & 35.41 & 81 \\
\hline AMOP2-S3 & 20/07 & 31/07 & 0.72 & 0.94 & 131 & 4.86 & 8.08 & 166 & 39.37 & 52.63 & 134 \\
\hline AMOP2-S4 & $31 / 07$ & $11 / 08$ & 0.3 & 0.28 & 95 & 1.75 & 2.02 & 115 & 4.01 & 10.26 & 256 \\
\hline AMOP2-S5 & $11 / 08$ & $22 / 08$ & 0.62 & 0.99 & 160 & 3.83 & 8.24 & 215 & 56.5 & 57.5 & 102 \\
\hline AMOP2-S6 & $22 / 08$ & $02 / 09$ & 0.8 & 0.96 & 120 & 5.32 & 7.69 & 145 & 77.26 & 51.67 & 67 \\
\hline AMOP2-S7 & $02 / 09$ & $13 / 09$ & 1.27 & 0.58 & 46 & 8.99 & 4.94 & 55 & 167.81 & 51.11 & 30 \\
\hline AMOP2-S8 & $13 / 09$ & $24 / 09$ & 0.93 & 0.45 & 49 & 6.4 & 3.42 & 53 & 115.31 & 39.96 & 35 \\
\hline AMOP2-S9 & 24/09 & $05 / 10$ & 0.63 & 0.8 & 127 & 4.91 & 6.46 & 132 & 100.85 & 27.84 & 28 \\
\hline AMOP2-S10 & $05 / 10$ & $16 / 10$ & 0.57 & 1.34 & 236 & 3.81 & 12.36 & 325 & 91.14 & 43.79 & 48 \\
\hline AMOP2-S11 & $16 / 10$ & $27 / 10$ & 0.47 & 0.33 & 70 & 2.71 & 1.95 & 72 & 42.71 & 2.66 & 6 \\
\hline AMOP2-S12 & $27 / 10$ & $07 / 11$ & 0.41 & 0.19 & 46 & 2.38 & 0.93 & 39 & 42.70 & 7.87 & 18 \\
\hline & Hig & eff & $\begin{array}{c}0.51 \\
( \pm 52 \%)\end{array}$ & $\begin{array}{c}\mathbf{0 . 6 4} \\
( \pm 57 \%)\end{array}$ & $\begin{array}{c}121 \\
( \pm 25 \%)\end{array}$ & $\begin{array}{c}3.72 \\
( \pm 46 \%)\end{array}$ & $\begin{array}{c}5.38 \\
( \pm 59 \%)\end{array}$ & $\begin{array}{c}138 \\
( \pm 38 \%)\end{array}$ & $\begin{array}{l}31.45 \\
( \pm 60 \%)\end{array}$ & $\begin{array}{l}39.01 \\
( \pm 45 \%)\end{array}$ & $\begin{array}{c}139 \\
( \pm 45 \%)\end{array}$ \\
\hline & Interm & te Teff & $\begin{array}{c}0.4 \\
( \pm 50 \%)\end{array}$ & $\begin{array}{c}0.44 \\
( \pm 54 \%)\end{array}$ & $\begin{array}{c}111 \\
( \pm 11 \%)\end{array}$ & $\begin{array}{c}3.36 \\
( \pm 54 \%)\end{array}$ & $\begin{array}{c}3.79 \\
( \pm 65 \%)\end{array}$ & $\begin{array}{c}113 \\
( \pm 19 \%)\end{array}$ & $\begin{array}{l}22.04 \\
( \pm 60 \%)\end{array}$ & $\begin{array}{l}24.63 \\
( \pm 58 \%)\end{array}$ & $\begin{array}{c}132 \\
( \pm 48 \%)\end{array}$ \\
\hline & Lo & eff & $\begin{array}{c}0.71 \\
( \pm 46 \%)\end{array}$ & $\begin{array}{c}0.62 \\
( \pm 67 \%)\end{array}$ & $\begin{array}{c}95 \\
( \pm 79 \%)\end{array}$ & $\begin{array}{c}4.87 \\
( \pm 51 \%)\end{array}$ & $\begin{array}{c}5.01 \\
( \pm 82 \%)\end{array}$ & $\begin{array}{c}113 \\
( \pm 97 \%)\end{array}$ & $\begin{array}{l}93.42 \\
( \pm 51 \%)\end{array}$ & $\begin{array}{l}28.87 \\
( \pm 69 \%)\end{array}$ & $\begin{array}{c}28 \\
( \pm 52 \%)\end{array}$ \\
\hline
\end{tabular}

Table S3b 


\begin{tabular}{|c|c|c|c|c|c|c|c|c|}
\hline \multirow{3}{*}{ Sample name } & \multicolumn{2}{|c|}{ Date in 2013} & \multirow{2}{*}{\multicolumn{2}{|c|}{$\begin{array}{c}\boldsymbol{\delta}^{13} \mathbf{C} \\
\% 0\end{array}$}} & \multirow{3}{*}{$\begin{array}{c}\text { Teff13C } \\
\%\end{array}$} & \multirow{2}{*}{\multicolumn{2}{|c|}{$\begin{array}{c}\boldsymbol{\delta}^{15} \mathbf{N} \\
\%\end{array}$}} & \multirow{3}{*}{$\begin{array}{c}\mathbf{T}_{\text {eff15N }} \\
\%\end{array}$} \\
\hline & \multirow[t]{2}{*}{ Start } & \multirow[t]{2}{*}{ End } & & & & & & \\
\hline & & & $34 m$ & $149 \mathrm{~m}$ & & $34 \mathrm{~m}$ & $149 \mathrm{~m}$ & \\
\hline AMOP1-S1 & $06 / 01$ & $13 / 01$ & -20.8 & -18.4 & 88 & 9,0 & 8.9 & 99 \\
\hline AMOP1-S2 & $13 / 01$ & $20 / 01$ & -20.3 & -18.8 & 93 & 6.6 & 8.4 & 127 \\
\hline AMOP1-S3 & $20 / 01$ & 27/01 & -20.3 & -19.1 & 94 & 5.9 & 8.6 & 146 \\
\hline AMOP1-S4 & $27 / 01$ & 03/02 & -20.3 & -19.3 & 95 & 3.5 & 9.3 & 267 \\
\hline AMOP1-S5 & 03/02 & $10 / 02$ & -21.0 & -19.8 & 94 & 5.5 & 10.1 & 185 \\
\hline AMOP1-S6 & $10 / 02$ & $17 / 02$ & -21.0 & -19.8 & 94 & 5.9 & 13.1 & 224 \\
\hline AMOP1-S7 & $17 / 02$ & $24 / 02$ & -21.0 & -20.8 & 99 & 9.2 & 8.0 & 86 \\
\hline AMOP1-S8 & $24 / 02$ & $03 / 03$ & -21.2 & -19.7 & 93 & 6.3 & 8.2 & 130 \\
\hline AMOP1-S9 & $03 / 03$ & $10 / 03$ & -21.5 & -20.2 & 94 & 5.9 & 8.0 & 135 \\
\hline AMOP1-S10 & $10 / 03$ & $17 / 03$ & -21.6 & -20.2 & 94 & 4.5 & 7.9 & 177 \\
\hline AMOP1-S11 & $17 / 03$ & $24 / 03$ & -21.1 & -19.3 & 91 & 7.9 & 9.1 & 116 \\
\hline AMOP1-S12 & $24 / 03$ & $31 / 03$ & -20.7 & -19.1 & 92 & 7.5 & 8.6 & 115 \\
\hline AMOP2-S1 & 28/06 & 09/07 & -22.0 & -21.0 & 95 & 4.3 & 4.3 & 100 \\
\hline AMOP2-S2 & 09/07 & 20/07 & -22.1 & -21.9 & 99 & 5.9 & 4.9 & 83 \\
\hline AMOP2-S3 & 20/07 & 31/07 & -22.7 & -21.9 & 96 & 7.7 & 6.9 & 89 \\
\hline AMOP2-S4 & 31/07 & $11 / 08$ & -22.3 & -20.5 & 92 & 9.3 & 8.0 & 86 \\
\hline AMOP2-S5 & $11 / 08$ & $22 / 08$ & -22.4 & -20.2 & 90 & 7.3 & 6.7 & 92 \\
\hline AMOP2-S6 & $22 / 08$ & 02/09 & -19.4 & -17.6 & 91 & 5.6 & 6.5 & 115 \\
\hline AMOP2-S7 & $02 / 09$ & $13 / 09$ & -19.4 & -17.4 & 90 & 4.9 & 7.4 & 152 \\
\hline AMOP2-S8 & $13 / 09$ & $24 / 09$ & -19.8 & -17.6 & 89 & 7.7 & 8.3 & 108 \\
\hline AMOP2-S9 & $24 / 09$ & $05 / 10$ & -18.4 & -17.8 & 97 & 6.9 & 6.8 & 98 \\
\hline AMOP2-S10 & $05 / 10$ & $16 / 10$ & -19.1 & -19.0 & 100 & 7.8 & 8.0 & 90 \\
\hline AMOP2-S11 & $16 / 10$ & $27 / 10$ & -19.7 & -19.9 & 101 & 9.3 & 8.4 & 90 \\
\hline AMOP2-S12 & $27 / 10$ & 07/11 & -20.4 & -21.4 & 105 & 7.9 & 8.1 & 103 \\
\hline & \multicolumn{2}{|c|}{ High Teff } & $\begin{array}{l}-21.2 \\
( \pm 5 \%)\end{array}$ & $\begin{array}{l}-19.8 \\
( \pm 6 \%)\end{array}$ & $\begin{array}{c}93 \\
( \pm 4 \%)\end{array}$ & $\begin{array}{c}6.7 \\
( \pm 13 \%)\end{array}$ & $\begin{array}{c}7.5 \\
( \pm 23 \%)\end{array}$ & $\begin{array}{c}113 \\
( \pm 23 \%)\end{array}$ \\
\hline & \multicolumn{2}{|c|}{ Intermediate Teff } & $\begin{array}{l}-21.2 \\
( \pm 3 \%)\end{array}$ & $\begin{array}{l}-19.9 \\
( \pm 3 \%)\end{array}$ & $\begin{array}{c}94 \\
(+2 \%)\end{array}$ & $\begin{array}{c}6.4 \\
(+31 \%)\end{array}$ & $\begin{array}{c}9.0 \\
(+19 \%)\end{array}$ & 156 \\
\hline & \multicolumn{2}{|c|}{ Low Teff } & $\begin{array}{r}-19.5 \\
( \pm 3 \%)\end{array}$ & $\begin{array}{r}-18.9 \\
( \pm 8 \%)\end{array}$ & $\begin{array}{c}97 \\
( \pm 6 \%)\end{array}$ & $\begin{array}{c}7.4 \\
( \pm 20 \%)\end{array}$ & $\begin{array}{c}7.7 \\
( \pm 9 \%)\end{array}$ & $\begin{array}{c}107 \\
( \pm 22 \%)\end{array}$ \\
\hline
\end{tabular}

Table S4 


\begin{tabular}{|c|c|c|c|c|c|}
\hline \multirow{3}{*}{ Sample name } & \multicolumn{2}{|c|}{ Date in 2013} & \multirow{2}{*}{\multicolumn{2}{|c|}{$\begin{array}{c}\mathrm{CaCO}_{3} \\
\mathbf{m g C a}^{-\mathbf{2}} \cdot \mathbf{d}^{\mathbf{- 1}}\end{array}$}} & \multirow{3}{*}{$\begin{array}{c}\mathrm{T}_{\text {effCaCO}} \\
\%\end{array}$} \\
\hline & \multirow[t]{2}{*}{ Start } & \multirow[t]{2}{*}{ End } & & & \\
\hline & & & $34 \mathrm{~m}$ & $149 \mathrm{~m}$ & \\
\hline AMOP1-S1 & $06 / 01$ & $13 / 01$ & 10.61 & 19.48 & 184 \\
\hline AMOP1-S2 & $13 / 01$ & $20 / 01$ & 9.92 & 13.22 & 133 \\
\hline AMOP1-S3 & $20 / 01$ & $27 / 01$ & 33.57 & 11.93 & 36 \\
\hline AMOP1-S4 & $27 / 01$ & $03 / 02$ & 24.27 & 4.54 & 19 \\
\hline AMOP1-S5 & $03 / 02$ & $10 / 02$ & 16.16 & 4.64 & 29 \\
\hline AMOP1-S6 & $10 / 02$ & $17 / 02$ & 19.25 & 3.73 & 19 \\
\hline AMOP1-S7 & $17 / 02$ & $24 / 02$ & 4.43 & 2.16 & 49 \\
\hline AMOP1-S8 & $24 / 02$ & $03 / 03$ & 10.16 & 2.11 & 21 \\
\hline AMOP1-S9 & $03 / 03$ & $10 / 03$ & 23.29 & 5.00 & 21 \\
\hline AMOP1-S10 & $10 / 03$ & $17 / 03$ & 9.82 & 7.04 & 72 \\
\hline AMOP1-S11 & $17 / 03$ & $24 / 03$ & 2.45 & 1.64 & 67 \\
\hline AMOP1-S12 & $24 / 03$ & $31 / 03$ & 6.29 & 2.06 & 33 \\
\hline AMOP2-S1 & $28 / 06$ & 09/07 & 2.46 & 14.54 & 591 \\
\hline AMOP2-S2 & 09/07 & $20 / 07$ & 4.39 & 5.82 & 133 \\
\hline AMOP2-S3 & $20 / 07$ & 31/07 & 0.63 & 6.14 & 969 \\
\hline AMOP2-S4 & 31/07 & $11 / 08$ & 17.00 & 1.28 & 8 \\
\hline AMOP2-S5 & 11/08 & $22 / 08$ & 0.70 & 1.87 & 267 \\
\hline AMOP2-S6 & $22 / 08$ & $02 / 09$ & 2.84 & 2.59 & 91 \\
\hline AMOP2-S7 & $02 / 09$ & $13 / 09$ & 373.38 & 2.02 & 1 \\
\hline AMOP2-S8 & $13 / 09$ & $24 / 09$ & 157.72 & 0.73 & 0 \\
\hline AMOP2-S9 & $24 / 09$ & $05 / 10$ & 49.12 & 1.05 & 2 \\
\hline AMOP2-S10 & $05 / 10$ & $16 / 10$ & 22.77 & 0.99 & 4 \\
\hline AMOP2-S11 & $16 / 10$ & $27 / 10$ & 14.95 & 4.78 & 32 \\
\hline AMOP2-S12 & $27 / 10$ & 07/11 & 25.32 & 0.96 & 4 \\
\hline & \multirow{2}{*}{\multicolumn{2}{|c|}{ High Teff }} & 10.21 & 6.9 & 127 \\
\hline & & & $( \pm 132 \%)$ & $( \pm 79 \%)$ & $( \pm 70 \%)$ \\
\hline & \multirow{2}{*}{\multicolumn{2}{|c|}{ Intermediate Teff }} & 14.52 & 3.62 & 30 \\
\hline & & & $( \pm 50 \%)$ & $( \pm 52 \%)$ & $( \pm 65 \%)$ \\
\hline & \multirow{2}{*}{\multicolumn{2}{|c|}{ Low Teff }} & 107.21 & 1.76 & 7 \\
\hline & & & $( \pm 131 \%)$ & $( \pm 88 \%)$ & $( \pm 170 \%)$ \\
\hline
\end{tabular}

Table S5 


\section{Figures}

Figure S1: Time series in 2013 for particle fluxes (left handed scale) at $34 \mathrm{~m}$ (black bar) and $149 \mathrm{~m}$ (white bar) and the corresponding transfer efficiency ( $T$; similarly to $T_{\text {eff }}$ defined from Eq.1, gray line, right-handed scale),

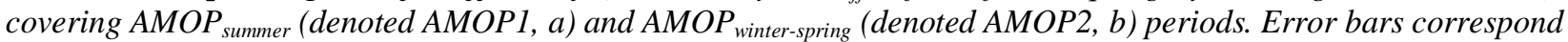
to the accuracy of analytical determination for the particle flux and is estimated through a logarithmic expansion of Eq. 1 for $T$ as for $T_{\text {eff. }}$ (cf. Methods, and Tab. S1 for details).

Figure S2: Relationship between the mass flux and the flux of carbon and nitrogen, and between their Transfer Ratios. a) Total mass fluxes versus the POC fluxes and b) total mass fluxes versus the PON fluxes, at $34 \mathrm{~m}$ (filled dots) and at $149 \mathrm{~m}$ (empty dots). c) Transfer efficiency of the total mass $(T)$ versus the transfer efficiency of the POC fluxes $\left(T_{\text {eff }}\right)$ and $\left.d\right)$ the transfer efficiency of the the total mass $(T)$ versus the transfer efficiency of the PON fluxes $\left(T_{\text {effPON }}\right)$. The color of the dots symbolizes the three different main $T_{\text {eff }}$ ranges (red, yellow and blue for low, intermediate and high $T_{\text {eff, }}$ respectively).

Figure S3: Vertical cross-shore section at $12^{\circ} \mathrm{S}$ at the latitude of the AMOP fixed mooring for the oxygen concentration and $\mathbf{p H}$ value. Data were acquired in the framework of $R / V$ L'Atalante AMOP cruise from January 27 to February 3, 2014. AMOP mooring location is indicated by the vertical white line near the coast on the right. The upper panel (a) represents the oxygen concentration ( $\left.\mu \mathrm{mol} . \mathrm{kg}^{-1}\right)$ and the lower panel (b) represents the pH values. On both graphs the white dashed isopleth corresponds to the averaged oxygen concentration at the depth where the oxygen gradient is the strongest over all the available AMOP cruise casts (lower oxycline boundary at $\mathrm{O}_{2}=87 \mu \mathrm{mol} . \mathrm{kg}^{-1}$ ) in $a-b$. The black isopleth corresponds to $\mathrm{pH}=7.6$ (average in the oxycline in $b$ ).

Figure S3: Transfer efficiency for the total mass fluxes and for Organic Matter. The black line represents the mass flux transfer efficiency $(T)$. The green line represents the transfer efficiency of the OM, where the OM is estimated as twice the POC fluxes according to Klaas and Archer (2002). Error bars are estimated from a logarithmic expansion of Eq. 1 and the analysis accuracies (cf. Methods), for both AMOP summer (denoted AMOP1) and AMOP winter-spring (denoted AMOP2) periods in 2013. 


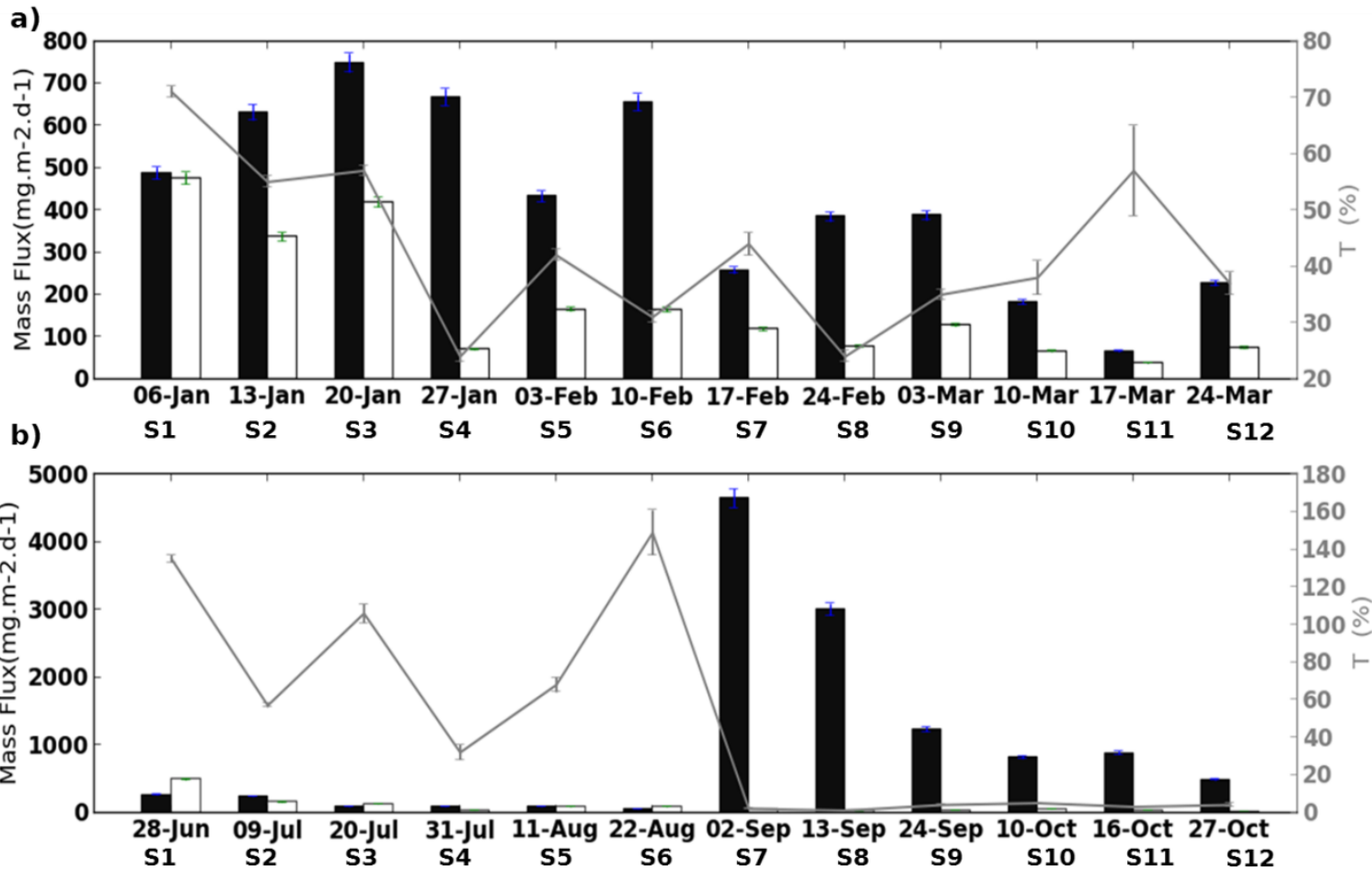

Figure S1 

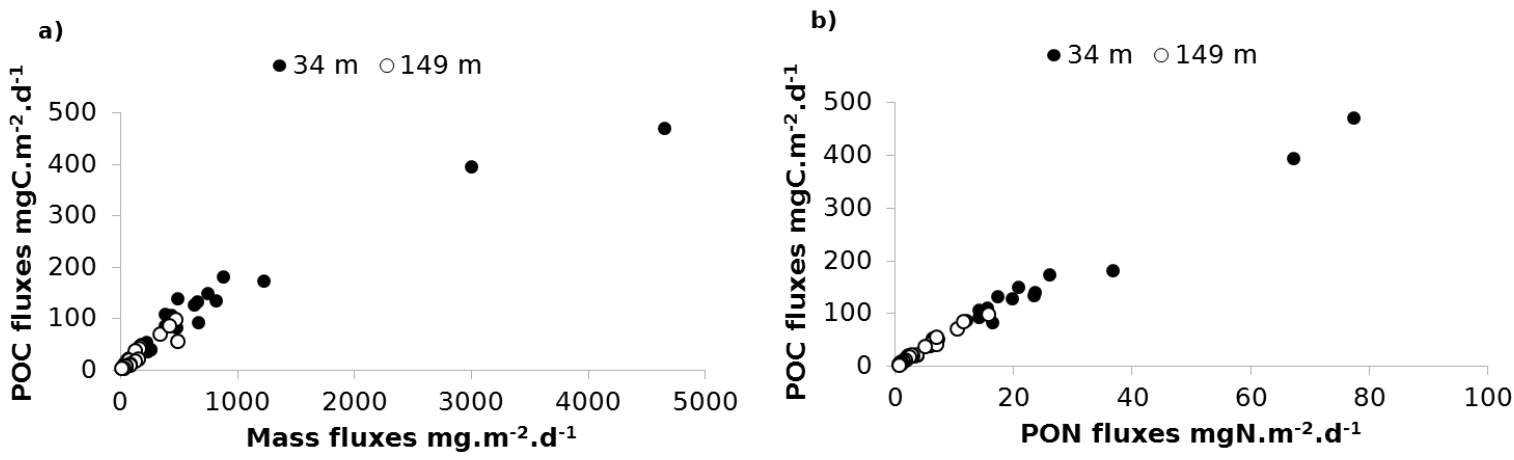

c) - High Teff Intermediate Teff • Low Teff

d)
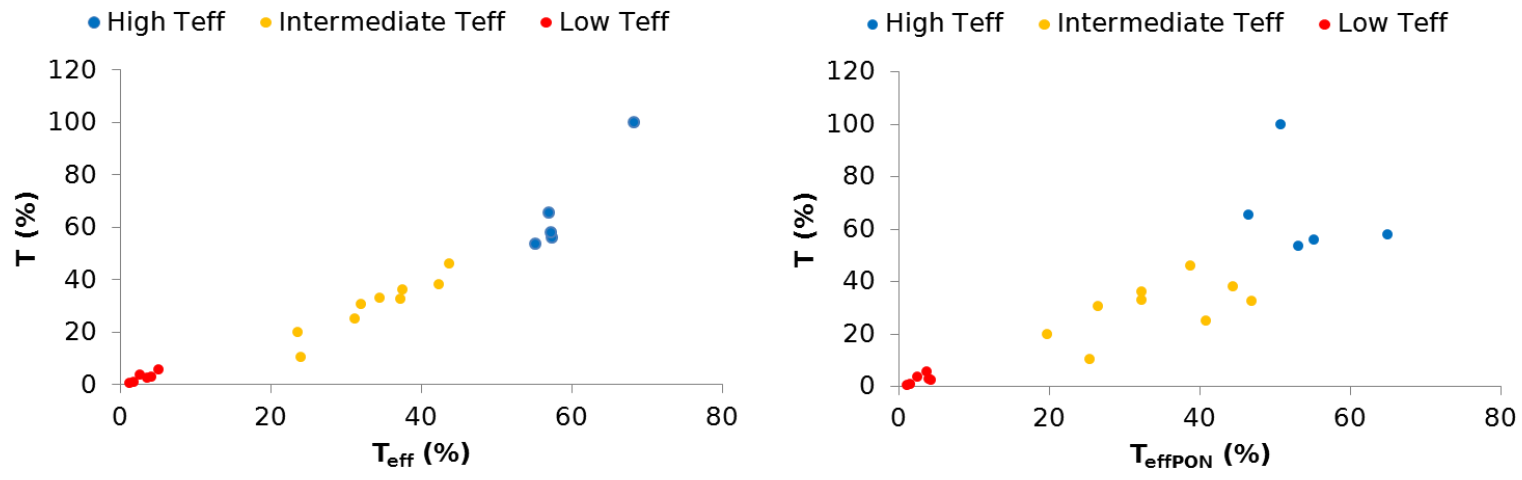

Figure S2 


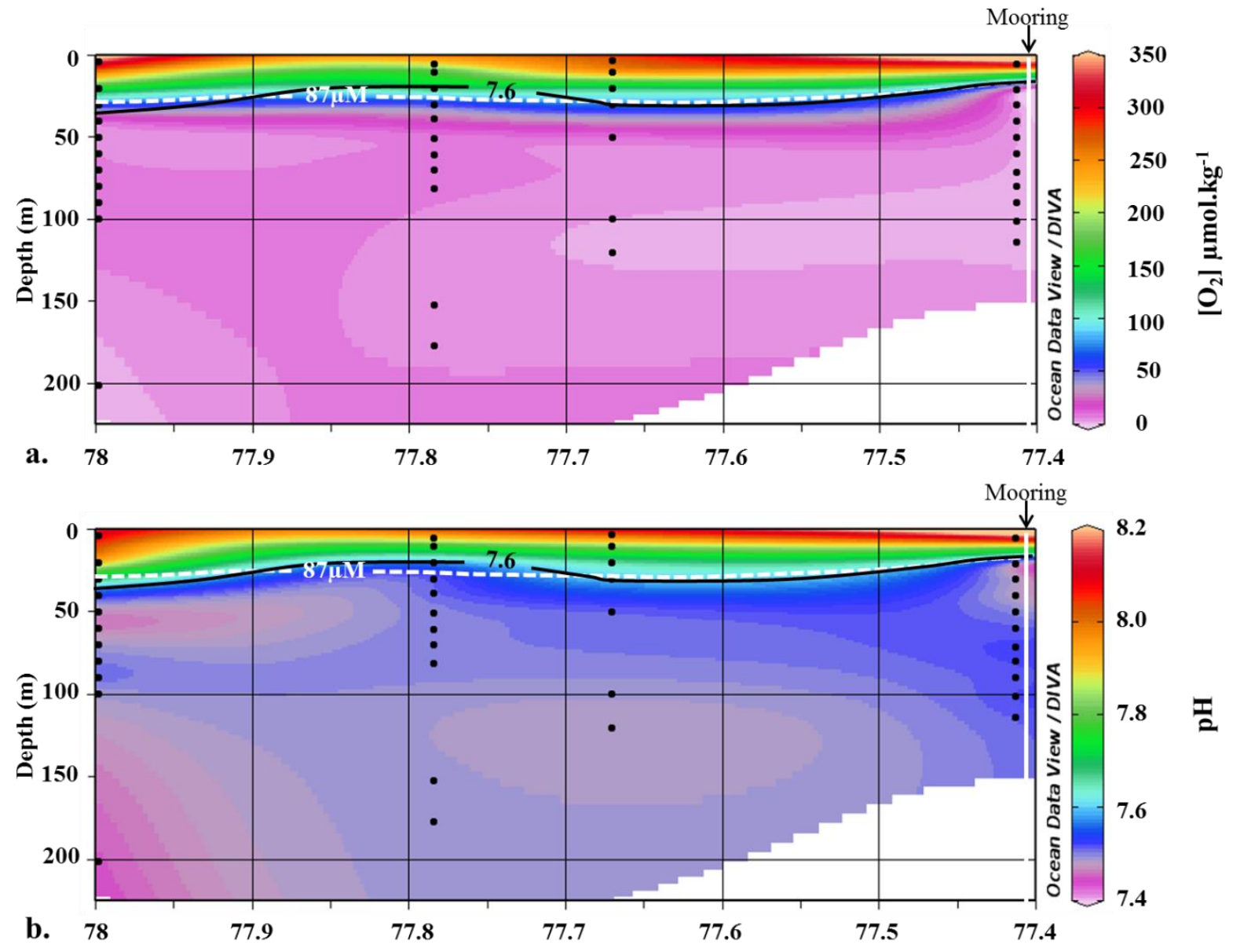

Figure S3 
a)
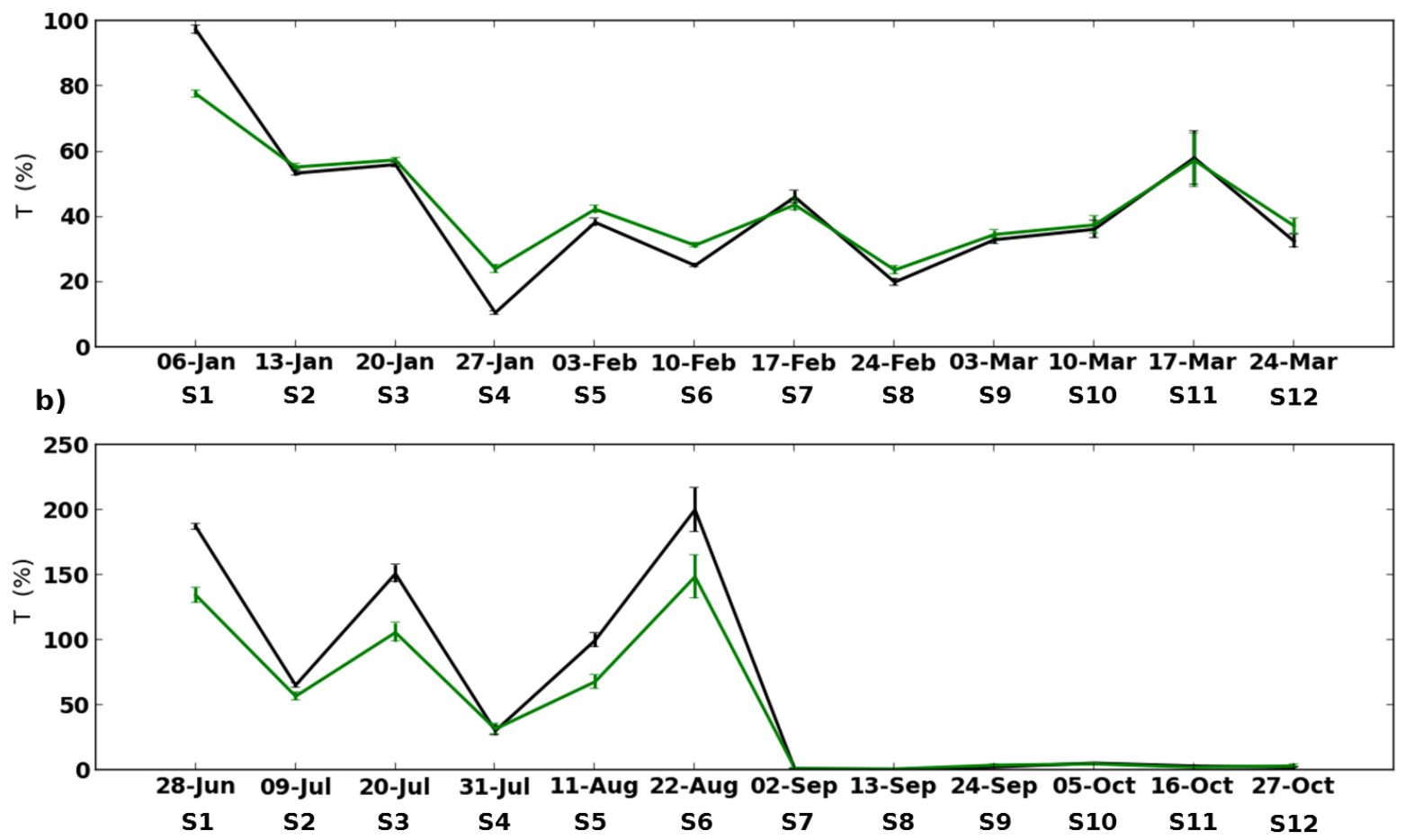

Figure S4 